\title{
Addressing the cultural complexity of OHS in the Australian mining industry ${ }^{1}$
}

\author{
Christine Aickin ${ }^{\mathrm{a}, 1}$, Andrea Shaw ${ }^{\mathrm{b}}$, Verna Blewett $^{\mathrm{c}}$, Laurie Stiller $^{\mathrm{d}}$, Stephen Cox $^{\mathrm{e}}$ \\ ${ }^{a}$ Workability Pty Ltd, 18 Pacific Street, Clovelly, NSW, Australia, 2031 \\ ' Shaw Idea Pty Ltd, 15 Fletcher Lane, Mt Egerton, Victoria, Australia,3352 \\ 'School of Psychology, Social Work and Social Policy, University of South Australia, Adelaide SA, Australia 5095 \\ ${ }^{\mathrm{d}}$ Beyond Words Pty Ltd, 2896 Oakey - Pittsworth Rd, Rossvale Queensland, Australia, 4356 \\ 'Stephen Cox Consulting Pty Ltd, 69 Whynot Street, West End, Queensland, Australia, Australia, 4101
}

\begin{abstract}
This paper summarises the findings of the site assessments of ten pilot mine sites involved in a project entitled, Creating a world-leading OHS culture in the NSW Mining Industry which was undertaken for the New South Wales Mine Safety Advisory Council (NSW MSAC). NSW MSAC was established in 1998 in NSW Australia and aims to increase the emphasis on safety and health within the mining industry by reviewing and analyzing safety performance, setting strategic directions, providing advice and developing policy recommendations. The project itself aimed to deliver a self-sustaining method for achieving and monitoring continuous improvement in OHS culture and practice to the NSW mining industry. The pilot sites involved in the project tested a set of self-assessment tools to enable mines to assess and improve their own OHS culture and performance on key elements of an OHS management system. The tools allowed examination of the current OHS culture of the sites. Sites then used a participative planning process to develop an improvement plan. This paper provides summary data only, without identifying the individual sites that were the source of the data.
\end{abstract}

Keywords: OHS culture, mining, OHS management systems, participative planning.

\section{Introduction}

This paper provides a summary of what was found in the site assessments of OHS Culture at ten sites across the mining industry in NSW, Australia. It does not provide a representative picture of the industry as these sites volunteered to participate. The extent to which they exemplify the circumstances across the industry is unknown.

The ten sites included one coal open cut, two coal underground, two metalliferous and two extractive clusters of mines (five mines in total).

Over the ten sites quantitative data was collected from 656 people via questionnaire, qualitative data from approximately 250 people via individual and focus group interviews and results from participative systems assessments.

\footnotetext{
${ }^{1}$ Corresponding author, E-mail: aickin@workability.com.au
}

\section{Methodology}

The methodology is based on the methods successfully used in the Digging Deeper project [1] in Australia and the Changing Minds, Changing Mines project [2] in South Africa.

The team included skilled interviewers and reviewers of organisations who were capable of collecting the data, skilled data analysts and experts in the areas under research in the mining and other industries in Australia and internationally. The collaboration of these individuals made it possible to successfully undertake the project.

Data collection tools included a site health and safety culture survey and a site OHS systems and practice self-assessment tool. 
A method to apply the data collection tools to produce an assessment of health and safety culture and systems was developed and piloted at the ten volunteer sites.

Finally, assistance was provided to the ten pilot sites to use the findings of the assessments to develop and implement action plans to address the opportunities identified in the assessments.

\section{Results}

\subsection{Culture}

The data collected on each site was used to provide a baseline assessment against the following seven (7) features of organisational culture:

- Mindfulness: International research into organisations that create reliable, safe workplaces suggests that such organisations create organisational "mindfulness", reflecting awareness of potential errors and orientation toward learning from these to minimize future risk. Mindfulness is thus a state of organisational readiness; being culturally and systematically ready to cope with the unexpected [3].

- Workgroup cohesion: describes the organisational capacity for peers to work together, rely on each other, ask for help, give health and safety a priority and, finally, to work safely without cutting corners even under pressure.

- Trust in management: represents a set of beliefs held by workers and others about the commitment management has to health and safety. It is demonstrated by the place OHS has in management decision-making, the provision of equipment and procedures to ensure health and safety, and in managerial competence with respect to OHS.

- Organisational justice: refers to the sense of fairness that exists at the workplace and the level of respect that is displayed for others. It also emphasizes diversity, elimination of discrimination and recognizes the societal impact of occupational health and safety.

- Supervisor support: is about the information and help provided by the supervisor or manager to their subordinates. Without supervisor support, addressing OHS problems becomes more difficult and communication channels become blocked and ineffective.
- Role clarity: means knowing what is expected of one in one's job, being given the information needed to do the job and being informed well in advance concerning changes or decisions affecting work.

- Work-life balance: means having sufficient time outside work time for family, friends, social life etc.

Each of the cultural features was scored on a scale of $1-5$, where 1 corresponds to strongly disagree and 5 corresponds to strongly agree. The average score for each feature is shown in Figure 1.

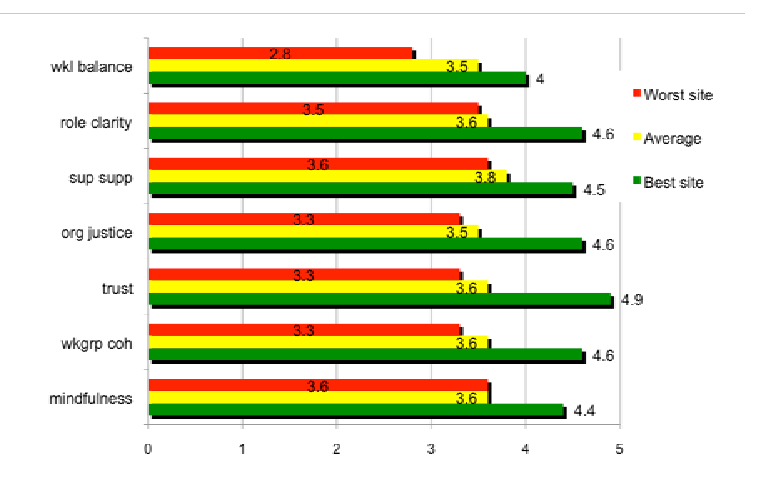

Figure1: Overall culture scores from questionnaire

As Figure 1 suggests, almost all pilot sites reported positively on all factors, with the most positive score on average being for supervisor support. There was one score below 3, for work-life balance at one site. There was some variation between sites, with a significant difference between the highest and lowest scores. Size of site was a key variable impacting on this difference.

This quantitative data was reinforced by the qualitative data collected at the sites, with the same variation between and within sites evident from the interview comments.

\subsection{Systems}

The following six OHS management system elements were defined for the project as the framework for providing an assessment of OHS management systems:

a) Role clarity and commitment

b) Consultation 
c) Risk management

d) Training and competence

e) Supervision

f) Evaluation and review

The following scoring system was used to selfassess these elements:

1. negative, ad hoc, non existent

2. reactive, inadequate

3. $\mathrm{OK}-$ meets the law

4. good-planned, proactive, preventive

5. fantastic - leads the industry and would be close to best practice outside the industry too.

Table 1 provides the average score given by the sites for each OHS management system element.

Table 1

Average score for system elements

\begin{tabular}{lc}
\hline \multicolumn{1}{c}{ System Element } & Average Score \\
\hline Risk management & 3.2 \\
\hline $\begin{array}{l}\text { Role clarity and com- } \\
\text { mitment }\end{array}$ & 3.3 \\
\hline Supervision & 3.1 \\
\hline Consultation & 3.2 \\
\hline Training & 2.7 \\
\hline Evaluation and review & 3.0 \\
\hline
\end{tabular}

Training received the lowest score. This resulted from the inclusion of non-technical skills training as a sub-element in this category. None of the sites had undertaken substantial work on this issue. Interestingly, all sites understood and had heard of the concept. They were also all considering or were interested in activities in this area.

Sites were able to use the scoring guide effectively. As the average scores set out above suggest, the process did not result in overly positive assessments.

The scoring systems identified that the reasons for undertaking each system element and the approach they were taking to the system were, on the whole, effective. Actually doing the work and, to a greater extent, reviewing and improving the system were not as effective.

\subsection{Future Inquiry Workshops}

Future Inquiry Workshops were run at each site. For the smaller sites, a modified workshop that took just over two hours was run. On other sites, a complete workshop over a full day was provided.

The process worked well where a full day workshop was run, with positive feedback from participants at the end of the workshop. For smaller sites, gaining full participation was challenging and at a couple of small sites, the small numbers meant that open and thorough discussion was difficult. Not surprisingly, the outcomes of these workshops may not have been as useful, although participants reported that the identified actions would not have been developed without the workshop. For a couple of the smaller sites, the workshop confirmed actions that were already underway.

The most common strategies were:

- Training (5 sites)

- Reporting and investigations (5 sites)

- Systems, including work procedures (4 sites)

- Communication (3 sites)

- Consultation (2 sites)

- Site infrastructure (2 sites)

Four sites identified specific risks that required more effective management action (eg fatigue, dust, housekeeping). Other sites identified issues associated with supervision and accountability, community relationships and contractor management as areas for work.

\section{Conclusion}

Most sites were already seeking to address the culture and systems issues covered in the project, which prompted their participation in the pilot. These sites, in particular, found the project a help in supporting and promoting action on identified issues. In general, participants on the pilot sites reported that the overall process was useful, leading to strategies that may not have been identified otherwise. Several of them were interested to continue to use the tools to monitor and review their progress. They also reported interest in participating in networking activities to share lessons and successes.

Common issues that we identified on the sites included the difficulty of balancing OHS and production effectively in the face of significant productivity pressure and flat management structures. Middle managers, in particular, have a considerable work- 
load and at some sites found it hard to participate in OHS consultative and planning activities. Their involvement in operational OHS matters was considerable, but they were sometimes playing a more reactive role because of lack of time to plan and participate. These latter activities were not treated as central to OHS on some sites.

As the results of the questionnaire suggest, worklife balance continues to challenge sites and is becoming a more overt issue. This relates both to shift work, but also to workload more generally for middle and senior managers.

While relationships appeared to be robust on most sites, it is difficult to be completely confident that people on small sites felt able to provide honest contributions during workshops and interviews. A number of steps were taken to provide some reassurance, but allowed participants to make their own decisions about how much they felt comfortable revealing. On a positive note, it is clear that the smaller sites in the pilot group had more effective consultation, leadership and communication, even if their management systems were less formal and they had less infrastructure resources.
Finally, this pilot process shows that the kit of draft assessment materials provides useful information that informed an effective planning process on the participating pilot sites. The method used were varied to suit the different sites with little loss of data integrity and relevant changes to the data collection and analysis tools were made as a result. Thus, the data collection method appears to be robust and reliable. When used by skilled facilitators, the pilot shows that the materials will achieve their aim of allowing sites to assess and improve their OHS culture and performance on key elements of an OHS management system and implement continuous improvement.

\section{References}

[1] A. Shaw, V. Blewett, L. Stiller, C. Aickin, S. Cox, S. Ferguson, and K. Frick, (2007), "Digging Deeper: Wran Consultancy Project Final Report". (Sydney: NSW Mine Safety Advisory Council).

[2] A. Shaw, V. Blewett, S. Cox, (2010), "Changing Minds, Changing Mines Final Project Report".

[3] K. Weick, and K. Sutcliffe (2007), Managing the Unexpected: Resilient Performance in an Age of Uncertainty (Second Edition; San Francisco: John Wiley \& Sons). 